\title{
Manipulating executive function with transcranial direct current stimulation
}

\author{
David V. Smith ${ }^{1,2 *}$ and John A. Clithero ${ }^{1,3}$ \\ 1 Center for Cognitive Neuroscience, Duke University, Durham, NC, USA \\ 2 Department of Psychology and Neuroscience, Duke University, Durham, NC, USA \\ ${ }^{3}$ Department of Economics, Duke University, Durham, NC, USA \\ *Correspondence: david.v.smith@duke.edu
}

\section{A commentary on}

Enhancement of planning ability by transcranial direct current stimulation

by Dockery, C. A., Hueckel-Weng, R., Birbaumer, N., and Plewnia, C. (2009). J. Neurosci. 29(22), 7271-7277.

Adaptive behavior frequently requires planning, or the ability to anticipate the consequences of actions and modify behavior accordingly. Situations that necessitate planning pervade our daily lives: engaging in strategic social interactions, determining the best route to an unfamiliar location, scheduling meetings in the midst of a hectic day, among many others. To succeed in these endeavors, one must calculate the potential repercussions of actions before proceeding along a specific course.

Within the laboratory, planning abilities and, more broadly, executive function, can be probed with the Tower of London (TOL) task. This task requires subjects to plan a series of moves that allow them to reach a predetermined goal configuration - the more moves required, the greater the cognitive challenge. Converging evidence has suggested that the dorsolateral prefrontal cortex (DLPFC) plays an important role in planning and executive function during the TOL task (e.g., Beauchamp et al., 2003).

Yet, the predominant trend in neuroscience studies of executive function has focused on neuroimaging or analogous techniques that involve measurements (or observations) of neural activity, often while ignoring other, less popular, techniques that entail manipulations of neural activity (Rorden and Karnath, 2004). Measurement techniques are correlative and do not afford the opportunity to examine whether specific parts of cortex are necessary for the execution of a specific task (Driver et al., 2009). In sharp contrast, manipulation techniques - cooling, lesions, transcranial magnetic stimulation (TMS), and transcranial direct current stimulation (tDCS) - allow one to test whether a specific part of the brain is necessary for a particular function. Nevertheless, the sheer popularity of measurement techniques compared to manipulation techniques is reflected in the disproportionate number of citations that neuroimaging studies receive (Fellows et al., 2005).

A recent report by Dockery et al. (2009) examines the effects of tDCS over the left DLPFC while subjects performed the TOL task. The usual setup for tDCS entails two large patch electrodes (generally $35 \mathrm{~cm}^{2}$ in size) placed on different parts of the scalp, with a current of around $1 \mathrm{~mA}$ flowing from the positively charged anodal electrode to the negatively charged cathodal electrode (Wagner et al., 2007). Compared to TMS and fMRI, the spatial resolution of tDCS is relatively poor given the large size of the electrodes (Wagner et al., 2007). In addition, the precise biophysical mechanisms underlying tDCS effects are unclear; but, it is believed that tDCS may exert its effects by modulating spontaneous background activity without necessarily evoking spikes directly like TMS (Wassermann and Grafman, 2005; Wagner et al., 2007). One well-established fact, however, is that placing the anodal electrode over a site of interest (i.e., anodal stimulation) increases cortical excitability, whereas placing the cathodal electrode over a site of interest (i.e., cathodal stimulation) decreases cortical excitability (Wagner et al., 2007). Because these polarity-dependent effects have been observed in isolated neurons (Terzuolo and Bullock, 1956), it has been suggested that they may rely (at least in part) on changes in neuronal membranes (Wassermann and Grafman, 2005).
Dockery et al. (2009) employed these differential effects of tDCS polarity over left DLPFC to measure manipulations of planning ability, as indexed by TOL task performance. They measured reaction time (RT) and accuracy across two levels of difficulty (low: one to two moves; high: four to five moves) as subjects received anodal, cathodal, or sham stimulation in a given experimental session. Critically, the order of the tDCS stimulation conditions was counterbalanced across subjects $(n=24)$ and the three experimental sessions, which were separated by an intersession interval of 1 week (Figure 1 of Dockery et al., 2009). In this multi-session approach, a given subject could receive anodal stimulation in the first session, sham stimulation in the second session, and cathodal stimulation in the third session. This design made it possible to test how $\mathrm{tDCS}$ polarity interacts with different stages of cognitive skill acquisition.

While there was a general effect for the more difficult task to be associated with increased RTs and lowered accuracy, there was no main effect of tDCS relative to sham stimulation on task performance (Figure 2 of Dockery et al., 2009). To test whether anodal and cathodal tDCS effects upon planning depend on learning phase, Dockery et al. (2009) first normalized accuracy and RT data to the sham condition within its respective session. Strikingly, tDCS polarity interacted with learning phase: cathodal stimulation yielded faster RTs relative to anodal stimulation in early sessions whereas anodal stimulation produced faster RTs and higher accuracy relative to cathodal stimulation in later sessions (Figure 4 of Dockery et al., 2009; note that "gain over sham (\%)" in the RT plots corresponds to faster, not increased, RTs for values exceeding 100\%). Moreover, these 
behavioral effects were observed up to a year later, suggesting a sustained effect on prefrontal cortex function.

These results demonstrate that both anodal and cathodal tDCS over left DLPFC can enhance planning abilities under certain conditions. Dockery et al. (2009) propose that cathodal tDCS acts as a "neuronal noise reducer" that aids in acquiring executive functions needed for performing the TOL in early sessions. In contrast, anodal stimulation only provides benefits when the subjects are already trained on the task. Noting previous work that links dopamine levels and stimulation effects (Kuo et al., 2008), Dockery et al. (2009) suggest that the differential effects of stimulation are mediated by the amount of available dopamine. In light of this interpretation, other studies (e.g., Fecteau et al., 2007) have found reductions in risk-taking behavior - a trait linked to dopamine (Schultz et al., 2008) - following tDCS over the DLPFC. Yet, if dopamine, as Dockery et al. (2009) suggest, plays an important role in tDCS modulations of planning abilities (and perhaps in tDCS modulations of risk preferences), then one could directly test the involvement of dopamine using positron emission tomography or pharmacological manipulations of dopamine. Under this model, one might expect to observe lower levels of dopamine in later stages of the learning phase when anodal tDCS improves performance.

However, the interaction found by Dockery et al. (2009) could also reflect an order effect of their experimental conditions. As a thought experiment, consider the possibility that early-cathodal tDCS improves performance on the TOL, with this enhancement carrying over to subsequent sessions. This effect would necessarily make early-anodal tDCS appear ineffectual and late-anodal tDCS appear beneficial because subjects who receive early-cathodal tDCS cannot receive early-anodal tDCS. This possibility could easily be explored by utilizing a between-subjects design (i.e., groups of sham, cathodal, anodal) rather than having a within-subject design (i.e., sessions of sham, cathodal, anodal). If tDCS effects upon planning are truly learning-phase dependent, then one would expect to see a similar interaction of stimulation and session when stimulation is manipulated between groups.
Along these same lines, a key component for all manipulation studies is well-matched control conditions. In TMS and tDCS studies, controls frequently entail "sham" stimulation in which the subject experiences the sensory effects of brain stimulation without invoking neural effects - cf. the approach taken by Dockery et al. (2009). Even without this control, though, subjects would not have any subjective insight into whether they were receiving anodal or cathodal stimulation. Dockery et al. (2009) correctly acknowledge that their results cannot conclusively implicate the left DLPFC; one alternative is opposite effects on the right frontal pole. To disambiguate these conflicting explanations, one would need to reverse the electrode locations (and Dockery et al., 2009 note that results from such an experiment are forthcoming). This additional experiment, though, would still not be sufficient to attribute the observed effects to a single part of left DLPFC; additional regions should be considered. One potential control site could be parietal cortex, which has been previously associated with the TOL task (Beauchamp et al., 2003; Boghi et al., 2006). Future studies will need to provide supporting evidence to clarify the role of left DLPFC in planning.

Identifying and testing control areas in stimulation studies can only partially rule out contributions from other brain areas. Concurrent stimulation and imaging studies have revealed distal effects of brain stimulation in remote areas (reviewed in Driver et al., 2009). Remote effects of brain stimulation can often vary as a function of context (Driver et al., 2009), suggesting that functional connections between regions are not fixed. In light of the design of Dockery et al. (2009), the application of tDCS could potentially affect remote brain areas (Lang et al., 2005), possibly even differentially across experimental sessions. Delineating whether (and how) other brain regions interact with tDCS over DLPFC will require additional studies of concurrent brain-stimulation and brain-imaging.

Neuroscience needs balance between techniques designed to test for correlation or causation; thus the challenges of interpreting tDCS and other manipulation techniques are welcome. Moreover, an often overlooked feature of manipulation studies involving tDCS (and TMS) is their potential for therapeutic intervention: these techniques can be used to improve function in individuals suffering from stroke, depression, and Parkinson's disease (Fregni and Pascual-Leone, 2007). While manipulation techniques offer the promise of helping individuals with cognitive deficits, recent work such as that by Dockery et al. (2009) suggests that manipulation methods can even enhance the abilities of neurologically healthy individuals. Specifically, they note that tDCS stimulation appeared to influence performance even up to a year after the stimulation and training - results that highlight the power of tDCS. These results can be extended in at least two important ways. First, the main effect of trainingdependent modulations should be applied to other paradigms that test executive function. Replicating the results of Dockery et al. (2009) in another domain (e.g., visuospatial attention) would provide an important first step in translating their results into a clinically efficacious therapy. Second, future research can examine individual differences in baseline executive functions (e.g., planning ability, risky decision making, among others) coupled with susceptibility to tDCS stimulation. As dopamine may mediate at least some of the behavioral effects of tDCS, studies could examine whether dopamine genetics distinguish differential responses to tDCS. Furthermore, although previous studies have documented gender differences in brain activation related to planning (Boghi et al., 2006), Dockery et al. (2009) did not have sufficient statistical power to investigate this issue. A natural supplement would include a larger and diversified sample that is amenable to exploring individual differences, both in gender and dopamine genetics. Given the dramatic and sustained effects of tDCS on executive function (Dockery et al., 2009), exploring these and other extensions may have immediate and broad clinical impact.

\section{ACKNOWLEDGMENTS}

We thank our mentor, Dr Scott Huettel, and DrChristopher Rorden for helpful comments and discussion regarding this paper. We also thank Drs McKell Carter and Richard Yaxley for feedback on earlier drafts. David V. Smith and John A. Clithero were supported by National Research Service Awards through NIMH grants F31MH086248 (DVS) and F31MH086255 (JAC). 


\section{REFERENCES}

Beauchamp, M. H., Dagher, A., Aston, J. A. D., and Doyon,J. (2003). Dynamic functional changes associated with cognitive skill learning of an adapted version of the Tower of London task. Neuroimage 20, 1649-1660.

Boghi, A., Rasetti, R., Avidano, F., Manzone, C., Orsi, L., D’Agata, F., Caroppo, P., Bergui, M., Rocca, P., Pulvirenti, L., Bradac, G. B., Bogetto, F., Mutani, R., and Mortara, P. (2006). The effect of gender on planning: an fMRI study using the Tower of London task. Neuroimage 33, 999-1010.

Dockery, C. A., Hueckel-Weng, R., Birbaumer, N., and Plewnia, C. (2009). Enhancement of planning ability by transcranial direct current stimulation. J. Neurosci. 29, 7271-7277.

Driver, J., Blankenburg, F., Bestmann, S., Vanduffel, W., and Ruff, C. C. (2009). Concurrent brain-stimulation and neuroimaging for studies of cognition. Trends Cogn. Sci. 13, 319-327.

Fecteau, S., Knoch, D., Fregni, F., Sultani, N., Boggio, P., and Pascual-Leone, A. (2007). Diminishing risktaking behavior by modulating activity in the prefrontal cortex: a direct current stimulation study. J. Neurosci. 27, 12500-12505.

Fellows, L. K., Heberlein, A. S., Morales, D. A., Shivde, G., Waller, S., and Wu, D. H. (2005). Method matters: an empirical study of impact in cognitive neuroscience. J. Cogn. Neurosci. 17, 850-858.

Fregni, F., and Pascual-Leone, A. (2007). Technology insight: Noninvasive brain stimulation in neurologyperspectives on the therapeutic potential of rTMS and tDCS. Nat. Clin. Pract. Neurol. 3, 383-393.

Kuo,M.-F., Paulus, W., and Nitsche, M.A. (2008). Boosting focally-induced brain plasticity by dopamine. Cereb. Cortex 18, 648-651.

Lang, N., Siebner, H. R., Ward, N. S., Lee, L., Nitsche, M. A. Paulus, W., Rothwell,J.C.,Lemon, R.N., and Frackowiak, R. S. (2005). How does transcranial DC stimulation of the primary motor cortex alter regional neuronal activity in the human brain? Eur. J. Neurosci. 22, 495.

Rorden, C., and Karnath, H.-O. (2004). Using human brain lesions to infer function: a relic from a past era in the fMRI age? Nat. Rev. Neurosci. 5, 812-819.

Schultz, W., Preuschoff, K., Camerer, C.,Hsu, M., Fiorillo, C. D., Tobler,P.N., and Bossaerts, P. (2008). Explicit neural signals reflecting reward uncertainty. Philos. Trans. $R$. Soc. Lond., B, Biol. Sci. 363, 3801-3811.

Terzuolo, C.A., and Bullock, T.H. (1956). Measurement of imposed voltage gradient adequate to modulate neuronal firing. Proc. Natil. Acad. Sci. USA 42, 687-694.

Wagner, T., Valero-Cabre, A., and Pascual-Leone, A. (2007). Noninvasive human brain stimulation. Annu. Rev. Biomed. Eng. 9, 527-565.

Wassermann, E. M., and Grafman, J. (2005). Recharging cognition with DC brain polarization. Trends Cogn Sci. 9, 503-505.

Received: 25 August 2009; published online: 08 October 2009.

Citation: Smith DV and Clithero JA (2009) Manipulating executive function with transcranial direct current stimulation. Front. Integr. Neurosci. 3:26. doi: 10.3389/neuro.07.026.2009

Copyright $(2009$ Smith and Clithero. This is an open-access article subject to an exclusive license agreement between the authors and the Frontiers Research Foundation, which permits unrestricted use, distribution, and reproduction in anymedium provided the original authors and source are credited. 